\title{
OBÉSITÉ
}

\section{Bernard Jeanrenaud}

Professeur ordinaire à la faculté de médecine de Genève, médecin, chef des laboratoires de recherches métaboliques.

\section{ADRESSE}

B. Jeanrenaud : laboratoires de recherches métaboliques, faculté et département de médecine, 64 avenue de la Roseraie, 1211 'obésité est devenue courante dans les sociétés industrialisées ou en voie d'industrialisation. Son existence indique que la régulation de l'équilibre énergétique est fréquemment imparfaite chez l'homme. L'obésité est due à une situation dans laquelle l'apport calorique excède son utilisation. Cependant, il est difficile d'établir lequel de ces deux paramètres prédomine quand le poids corporel excède la normale. Bien que l'hyperphagie soit souvent incriminée en tant que cause primaire de l'obésité - et ceci est parfois le cas - la plupart des enquêtes diététiques se solde par un échec lorsque l'on cherche à prouver que l'hyperphagie joue effectivement un rôle décisif : il est donc possible que l'hyperphagie (relative ou absolue) soit plutôt un facteur d'aggravation qu'un facteur causal de l'obésité. L'intérêt relativement récent pour cette maladie fait suite à des décades d'un désintérêt considérable, tant sur le plan scientifique qu'humain. Des études nombreuses ont été finalement entreprises, surtout lorsque l'on constata que l'obésité se soldait par des complications sérieuses telles que le diabète, des hyperlipémies, une pathologie athéromateuse menant à des désordres cardio-vasculaires, de l'hypertension, des affections pulmonaires, des dégénérescences produisant des troubles de la vision, des affections tant rénales que biliaires. L'intérêt médical et scientifique qu'a suscité l'obésité est aussi à mettre en relation avec un succès thérapeutique relativement médiocre dans cette maladie. Il est illustré enfin par la création récente de nombreuses sociétés savantes dont le but est de mieux comprendre la physiopathologie de ce syndrome. De telles sociétés sont actives aux ÉtatsUnis, ainsi que dans de nombreux pays européens. En France, elles sont représentées par l'Association
RÉFÉRENCES

1. Björntorp $P$, Cairella $M$, Howard AN. Recent Advances in Obesity Research: III. London : John Libbey, 1980.

2. Björntorp $P$, Karlsson $M$, Pettersson P, Sypniewska G. Differentiation and function of rat adipocyte precursor cells in primary culture. J Lipid Res 1980 ; 21 : 714-23.

3. Czech MP. The nature and regulation of the insulin receptor: structure and function. Annu Rev Physiol 1985; 47 : 357-81.

4. Girardier L, Stock MJ. Mammalian Thermogenesis. London, New York : Chapman and Hall, 1983.

5. Grimaldi $P$, Djian $P$, Négrel $R$, Ailhaud G. Differentiation of Ob17 preadipocytes to adipocytes : requirements of adipose conversion factor(s) for fat cell cluster formation. $E M B O J$ $1982 ; 6$ : 687-92.

6. Rohner-Jeanrenaud F, Bobbioni E, Ionescu E, Sauter JF, Jeanrenaud B. Central nervous system regulation of insulin secretion. Adv Metab Disord 1983 ; 10 : 193-220.

$\mathrm{m} / \mathrm{s} n^{\circ} 7 \mathrm{vol} .3$, septembre 87 
française pour l'étude et la recherche sur l'obésité, l'AFERO. Le traitement de l'obésité est un défi majeur tant pour les médecins, les physiologistes, les nutritionnistes que pour les chercheurs. L'obtention d'une diminution de poids, grâce à une prise en charge médicale et psychologique, est souvent un succès à court terme, qui contraste avec le faible pourcentage des succès thérapeutiques à long terme.

Les raisons sous-jacentes à ces échecs sont, sinon expliquées, tout au moins suggérées par les articles que l'on lira dans ce numéro.

Le Docteur Gérard Ailhaud expose les mécanismes de la multiplication et de la différenciation des cellules adipeuses. On y voit les mécanismes complexes (stimuli mitogènes et de différenciation adipocytaire) par lesquels est régulée la multiplication des cellules adipeuses ainsi que les cellules précurseurs de celles-ci. On y voit que certains de ces stimuli déterminent le nombre final de cellules adipeuses qu'un individu aura durant sa vie. Ces cellules se rempliront plus ou moins de lipides, mais leur nombre ne se modifiera guère. Ainsi, le nombre total de cellules adipeuses que possède un individu donné est l'une des raisons pour lesquelles, après une perte de poids vidant les cellules adipeuses de leurs excès lipidiques, ces dernières sont capables de se remplir à nouveau. Ceci pourrait expliquer les échecs d'une thérapeutique lorsqu'elle est interrompue.

L'article du Docteur Josiane Seydoux montre les relations qui existent entre l'obésité et les défauts de la thermogenèse, c'est-à-dire la propriété qu'a l'organisme de dissiper l'énergie sous la forme de chaleur, un processus qui est sous le contrôle du système sympathique. Il illustre le fait que, dans beaucoup de modèles animaux $\mathrm{m} / \mathrm{s} n^{\circ} 7$ vol. 3 , septembre 87 d'obésité, il existe un déficit de la thermogenèse. Il souligne que, tant chez l'animal que chez l'homme, il existe une protéine découplante qui est responsable de cette thermogenèse ; chez l'homme, son rôle précis reste à démontrer et son effet pourrait être complété par une thermogenèse due à la musculature.

Le texte du Docteur Yannick Le Marchand-Brustel illustre comment s'établit, chez l'obèse, un état de résistance à l'action de l'insuline. Un volet de cet article porte sur la définition même de l'insulinorésistance. Il donne ensuite des exemples des défauts qui s'établissent aux différentes étapes de l'action de l'insuline et qui sont responsables de l'insulinorésistance. Des altérations existent au niveau même de l'interaction de l'insuline avec son récepteur membranaire. D'autres altérations se situent au niveau d'enzymes intracellulaires et de leurs fonctions respectives. Ce texte illustre ainsi la notion d'une double pathologie, au niveau des récepteurs d'une part, à des étapes postérieures à ces récepteurs d'autre part. L'article montre que l'obésité est un syndrome évolutif et que l'obésité d'installation récente est différente d'une obésité ancienne. Il illustre le rôle que joue l'hyperinsulinémie dans l'établissement même de l'insulinorésistance.

L'article du Docteur Bernard Jeanrenaud tente de suggérer que certains types d'obésité auraient pour origine un dysfonctionnement du système nerveux autonome. $\mathrm{Ce}$ dysfonctionnement se caractériserait par une augmentation de certaines efférences vagales se rendant, en particulier, au pancréas endocrine, expliquant par là l'apparition d'une augmentation de la sécrétion d'insuline. Ce dysfonctionnement comprendrait également une diminution de certaines efférences sympathiques allant de l'encéphale vers la périphérie, altération compatible avec les défauts de la thermogenèse. Ces dysfonctionnements initiaux puis persistants du système nerveux autonome expliqueraient (en particulier par le biais de l'hyperinsulinémie) l'installation de l'obésité dont la réversibilité serait difficile non seulement pour les raisons évoquées par le Dr. Gérard Ailhaud, mais également parce que les voies lipogéniques ne semblent pas devenir insulinorésistantes. Cet article illustre le caractère multifactoriel des anomalies de l'obésité présentée comme une affection neuroendocrinienne dans laquelle, en ce qui concerne l'évolution vers le diabète de type 2 , l'hyperinsulinémie ainsi que des anomalies de la sécrétion des hormones de contrerégulation de l'insuline (glucagon, catécholamines, glucocorticoïdes) jouent un rôle dont l'importance respective reste à déterminer.

Comme on le verra, les articles sus-mentionnés sont basés sur des expériences réalisées in vitro et presque toujours sur des modèles animaux. L'extrapolation à la pathologie humaine est ainsi difficile. L'homme obèse possède probablement, à des degrés variables, certains des éléments décrits chez l'animal. Ceci explique peutêtre le caractère hétérogène des obésités humaines.

Il est impossible de prédire l'avenir. On peut cependant penser que l'interpénétration des données présentées dans ces articles de synthèse et de celles (passées, présentes et futures) obtenues en clinique, aboutira à une modification de notre perception actuelle de l'obésité humaine. Cela pourrait conduire à des approches thérapeutiques différentes et plus performantes que celles utilisées aujourd'hui 